\title{
Concepciones y Prácticas de Enseñanza Declaradas sobre Aspectos Sociológicos de la Naturaleza de la Ciencia de Docentes de Escuela Secundaria de una Ciudad de Argentina
}

\section{Conceptions and Declared Teaching Practices about Sociological Aspects of the Nature of Science of High School Teachers from a City in Argentina}

\author{
iD) Iván Felsztyna \\ Daniela Silvina Arán² \\ Leticia Garcia Romano ${ }^{1}$

\begin{abstract}
'Universidad Nacional de Córdoba, Facultad de Ciencias Exactas, Físicas y Naturales, Departamento de Enseñanza de la Ciencia y la Tecnología, IIByT, CONICET-UNC, Córdoba, Argentina.

Autor correspondiente: ivan.felsztyna@unc.edu.ar

${ }^{2}$ Universidad Nacional de Córdoba, Instituto Multidisciplinario de Biología Vegetal, Consejo Nacional de Investigaciones Científicas y Técnicas, Córdoba, Argentina.
\end{abstract}

Resumen: Los contenidos de naturaleza de la ciencia (NOS, por sus siglas en inglés) se pueden clasificar en tres ejes: epistemológico, histórico y sociológico. La investigación didáctica ha priorizado el eje epistemológico y persiste un debate sobre qué contenidos de NOS enseñar y cómo hacerlo. El objetivo del trabajo es analizar las concepciones y prácticas de enseñanza declaradas sobre aspectos sociológicos de la NOS en docentes de Metodología de la Investigación en Ciencias Naturales de la ciudad de Córdoba, Argentina. Se utilizó una metodología cualitativa con entrevistas semiestructuradas. Los docentes entrevistados/as enfatizan la enseñanza explícita del eje epistemológico. Reconocen las influencias mutuas entre ciencia y sociedad, aunque persisten algunas concepciones ingenuas, particularmente respecto a la imagen del científico/a. En cuanto a las prácticas de enseñanza, se evidenció que las temáticas sociológicas se trabajan en el aula, a veces de manera planificada y otras de manera espontánea.

Palabras-clave: Naturaleza de la ciencia; Sociología de la ciencia; Metodología de la investigación; Enseñanza de las ciencias naturales; Prácticas de enseñanza.

Abstract: The contents of Nature of Science (NOS) can be classified in the epistemological, historical and sociological perspectives. The research on didactics of science has prioritized the epistemological perspective, and a debate persists among specialists about which contents of NOS to teach and how to do it. The aim of this research is to analyze the conceptions and declared practices of teaching about sociological aspects of NOS among teachers of Methods in Research for Natural Sciences in the city of Córdoba, Argentina. A qualitative methodology was used with semi-structured interviews. The interviewed teachers emphasize the explicit teaching of the epistemological perspective of NOS. They recognize mutual influences between science and society although some naive conceptions persist, particularly about the image of the scientist. Regarding teaching practices, it was evident that sociological issues are developed in the classrooms; sometimes planned and sometimes spontaneously.

Keywords: Nature of science; Sociology of science; Methods in research; Natural sciences teaching; Teaching practices.

Recebido em: 20/07/2020

Aprovado em: 21/12/2020 


\section{Introducción}

La didáctica de las ciencias considera actualmente que el aprendizaje de la naturaleza de la ciencia (NOS, por las siglas en inglés de nature of science) es uno de los dos componentes esenciales de la alfabetización científico-tecnológica, junto con el conocimiento de los conceptos de la ciencia y la tecnología propiamente dichos. Se sostiene, entonces, que la reflexión crítica acerca de qué es la ciencia, cómo se elabora y cómo se relaciona con la sociedad, es tan importante para la formación de los ciudadanos como el hecho de conocer los propios contenidos científicos (BENNÁSSAR ROIG et al., 2010; LOZANO; BAHAMONDE; ADÚRIZ-BRAVO, 2016).

Se puede definir a la NOS como un conjunto de metaconocimientos sobre la ciencia que trata acerca de la construcción, el establecimiento y la organización del conocimiento científico (MOURA, 2014). Estos metaconocimientos surgen a partir de reflexiones interdisciplinarias de expertos/as en filosofía, historia y sociología de la ciencia, así como de científicos/as y educadores/as. (DAGHER; ERDURAN, 2016).

Los aportes de la NOS a la enseñanza de las ciencias naturales pueden clasificarse en tres grandes campos temáticos, que se corresponden con una serie de preguntas que podemos formularnos acerca de la ciencia:

(1) El eje epistemológico apunta a determinar qué es la ciencia y cómo se elabora,

(2) el eje histórico intenta responder a la pregunta de cómo cambia la ciencia en el tiempo, (3) el eje sociológico quiere caracterizar la cuestión de cómo se relaciona la ciencia con la sociedad y la cultura (ADÚRIZ-BRAVO, 2005, p. 4-5).

Por otra parte, la diversidad de estrategias de enseñanza de los contenidos de NOS suele clasificarse en dos enfoques básicos. Por un lado, el enfoque implícito considera que el aprendizaje de la NOS se da de manera indirecta, a través de las actividades usuales de enseñanza de la ciencia. Por otro lado, el enfoque de tipo explícito incluye los contenidos de la NOS en la actividad docente, lo cual implica algún tipo de planificación curricular y significativa de objetivos, contenidos, evaluación y realización en el aula (BENNÁSSAR ROIG et al., 2010).

A pesar de que existe un consenso acerca de la enorme relevancia del aprendizaje de la NOS, su carácter multifacético y dinámico hace difícil determinar cuáles son los conocimientos concretos que deben ser enseñados dentro de este campo (ACEVEDO DÍAZ; GARCÍA-CARMONA, 2016). El debate al respecto, aún no saldado, ha dado lugar a diferentes perspectivas teóricas (LOZANO; BAHAMONDE; ADÚRIZ-BRAVO, 2016).

Moura (2014) realiza una revisión bibliográfica acerca de esta discusión, clasificando las ideas de los investigadores en el área de la NOS en dos posiciones diferenciadas. Por un lado, se encuentran quienes sostienen que aquello que se debe enseñar es una serie de aspectos consensuales. Esta perspectiva pretende constituir una lista de afirmaciones generalizables, simples y objetivas, que definirían aquello que está involucrado en la construcción del conocimiento científico. Por otro lado, se ubican quienes critican esta denominada "visión de consenso", sosteniendo concepciones más amplias y relacionales, tal como la perspectiva de parecido de familia ("family resemblance") (IRZIK; NOLA, 2011).

El enfoque de parecido de familia sostiene que las características de la ciencia no muestran relaciones de identidad total entre las diversas disciplinas, sino más bien 
de semejanza entre ellas, de una manera análoga a los rasgos comunes compartidos entre los distintos miembros de una familia. Este parecido común entre las ciencias se categoriza dentro de dos dimensiones: epistémico-cognitiva y social-institucional. La segunda dimensión abarca aspectos tales como las actividades profesionales de los científicos, la crítica científica, la certificación y diseminación social del conocimiento científico y los valores institucionales y sociales de la ciencia (ERDURAN; DAGHER, 2014; IRZIK; NOLA, 2014).

La perspectiva de Allchin (2013) acerca de la NOS, denominada "ciencia integral" ("whole science"), también se considera como una alternativa superadora de la visión de consenso (BEJARANO; ADÚRIZ-BRAVO; BONFIM, 2019). Esta propuesta incluye tres dimensiones del conocimiento científico: observacional, conceptual y socio-cultural. Esta última incluye aspectos tales como las instituciones científicas, las fuentes de financiamiento, las normas de conducta de la ciencia, la comunicación científica y el papel de la ideología, la religión y la nacionalidad en la construcción del conocimiento (ALLCHIN, 2013).

Puede observarse que estas perspectivas más actualizadas y holísticas otorgan un papel significativo a los aspectos de la sociología externa e interna de la ciencia dentro de la NOS. Con "sociología externa de la ciencia" nos referimos a las influencias mutuas entre la sociedad y el sistema científico-tecnológico, la influencia simultánea entre la ciencia, la tecnología y la sociedad (CTS) y a la influencia de la educación científica. Por su parte, la "sociología interna de la ciencia" comprende las características personales de los científicos y las científicas, como así también la construcción social de la ciencia y la tecnología (VÁZQUEZ ALONSO; MANASSERO MAS, 2015). En este sentido, estas propuestas teóricas contrastan con la visión de consenso, en la cual las relaciones de la ciencia con la sociedad y la cultura ocupan un lugar secundario frente a la enseñanza de los contenidos estrictamente epistemológicos.

En relación a esto, algunos autores reflexionan acerca de la relevancia de los aspectos sociológicos en la enseñanza de la NOS. Zemplén (2007) argumenta en favor de colocar los aspectos sociológicos en el centro de esta temática. Según este autor, la perspectiva sociológica promueve la superación de ciertos problemas conceptuales que suelen surgir en las currículas de NOS actualmente dominantes. Asimismo, menciona que un punto de partida sociológico para la NOS presenta una visión más realista de la ciencia y resulta más cercano a la vida cotidiana del estudiantado, en comparación al clásico recorrido inductivista-falsacionista-kuhniano de los diseños de enseñanza estrictamente epistemológicos. Aún así, el objetivo de la priorización del eje sociológico no consiste en reemplazar los aspectos epistémicos, sino en proporcionar un marco que les otorgue a estos contenidos mayor sentido y una contextualización más clara en la enseñanza de la NOS. Por otra parte, para Farías (2012), dado que la ciencia es una actividad humana e íntimamente ligada a la cultura que la produce, la mirada sociológica debe ser incluida en la enseñanza de la NOS, de manera tal que el estudio de la ciencia sea más democrático. Aun así, existe una mirada reticente de parte de la didáctica de las ciencias hacia los trabajos provenientes de la sociología de la ciencia.

En este sentido, el predominio del eje epistemológico se encuentra reflejado en el recorrido de las líneas de investigación en el campo de la NOS. Dagher y Erduran (2016) destacan que la visión de consenso condujo a un gran número de estudios empíricos sobre las concepciones epistemológicas de estudiantes y docentes. Sin embargo, las 
concepciones y las prácticas de enseñanza de los aspectos sociológicos de la NOS han recibido menor atención.

Un primer hito en cuanto a los instrumentos metodológicos para el estudio de concepciones sociológicas de la NOS está marcado por el cuestionario Views on ScienceTechnology-Society (VOSTS) (AIKENHEAD, 1992). Posteriormente, a finales de la década de 1990, un grupo de investigadores españoles desarrolla el Cuestionario de Opiniones sobre Ciencia, Tecnología y Sociedad (COCTS) (VÁZQUEZ ALONSO, et al., 2006). Los contenidos del COCTS incluyen definiciones y relaciones de ciencia y tecnología, epistemología, relaciones CTS, educación en ciencia y tecnología, y sociología interna de la ciencia. Este cuestionario fue utilizado como instrumento en muchas investigaciones, entre las cuales se destaca el Proyecto Iberoamericano de Evaluación de Actitudes Relacionadas con la Ciencia, la Tecnología y la Sociedad (PIEARCTS) (BENNÀSSAR ROIG et al., 2010).

Diversos autores destacan la relevancia del rol del docente en la enseñanza de la NOS y el papel que juegan sus propias concepciones en el proceso de construcción de conocimientos metacientíficos por parte de los y las estudiantes (VÁZQUEZ ALONSO; MANASSERO MAS, 2016). Teófilo y Gallão (2019) consideran que, en el marco de los debates acerca de la NOS, el docente se constituye en el protagonista de un proceso de enseñanza que debe evitar la mera propagación de información, sin la debida reflexión y realización de cuestionamientos.

En este marco, tomamos como antecedentes algunos estudios que buscaron indagar las concepciones de docentes acerca de aspectos sociales de la ciencia. En particular, en el ámbito latinoamericano, encontramos el trabajo de Auler y Delizoicov (2006), quienes realizaron entrevistas semi-estructuradas acerca de asuntos socio-científicos a un grupo de docentes de ciencias en Santa María, Brasil, con el fin de analizar sus ideas sobre las relaciones CTS. También resulta de interés el trabajo de Miranda y Freitas (2008), en el cual se indagan las concepciones de un grupo de docentes de ciencias en São Carlos, por medio de la aplicación del cuestionario VOSTS y de entrevistas semi-estructuradas. Por otra parte, Kist y Ferraz (2010), realizaron entrevistas estructuradas a docentes de biología de la región de Paraná, Brasil, con el objetivo de indagar su comprensión acerca de las relaciones CTS, como así también sus prácticas de enseñanza declaradas respecto a dichos contenidos. Antonioli et al. (2012) utilizaron el COCTS con la finalidad de analizar las actitudes para el aprendizaje de la ciencia de profesores y estudiantes universitarios en Río de Janeiro. Sepini y Maciel (2016) utilizaron el mismo instrumento para evaluar los cambios en las concepciones de la NOS luego de la aplicación de un curso sobre esta temática a un grupo de docentes en formación. En el caso de Argentina, encontramos el trabajo de Pujalte, Adúriz-Bravo y Porro (2014), quienes realizaron cuestionarios a un grupo de profesores de Biología sobre su imagen de ciencia y sobre la enseñanza de las ciencias. Este estudio incluyó la realización de entrevistas y observaciones de clase a algunos de los docentes previamente encuestados, abordando aspectos principalmente epistemológicos.

Finalmente, es importante revisar qué proponen los Diseños Curriculares de la Provincia de Córdoba, Argentina, respecto a los contenidos de NOS. Para el Ciclo Orientado en Ciencias Naturales se sostiene que el tratamiento de temáticas científicas relevantes desde miradas actualizadas "[...] pone en evidencia a la ciencia en su carácter de producción humana, cultural y social, revisable, históricamente situada y atravesada 
por las mismas complejidades e intereses que caracterizan a la sociedad en la que se desarrollan" (CÓRDOBA, 2012). Además, dentro de los Espacios de Orientación Institucional propuestos se incluyen tres asignaturas estrechamente vinculadas a estas temáticas. Una de ellas se denomina Metodología de la Investigación en Ciencias Naturales (MICN) y es la que habitualmente se encuentra incluida en las escuelas secundarias de la ciudad de Córdoba con Ciclo Orientado en Ciencias Naturales. En el diseño curricular se sugiere que en MICN se aborden los procesos de investigación científica en el campo de las ciencias naturales, la tarea del investigador como parte de la comunidad científica, la actitud científica y la comunicación en ciencia (CÓRDOBA, 2012).

Con base en lo expuesto anteriormente, en este trabajo nos proponemos como objetivo explorar tanto las concepciones como las prácticas de enseñanza declaradas acerca de aspectos sociológicos de la NOS en docentes del Espacio de Orientación Institucional MICN de escuelas de la ciudad de Córdoba. Los estudios acerca de estas temáticas son escasos para la ciudad y nuestro país, por lo cual esta indagación resulta de gran valor en este contexto.

\section{Metodología}

Se utilizó una metodología cualitativa, que consistió en la realización de entrevistas semiestructuradas a un profesor y tres profesoras de secundaria del espacio curricular Metodología de la Investigación en Ciencias Naturales (MICN) de cuatro escuelas diferentes, en la ciudad de Córdoba (Cuadro 1). Una de las ventajas de utilizar la modalidad semiestructurada para la realización de entrevistas es la posibilidad de adaptarse a los sujetos, explorar aspectos vinculados a sus sentimientos y valores, aclarar términos, identificar ambigüedades y reducir formalismos (DÍAZ-BRAVO et al., 2013; MIRANDA; FREITAS, 2008).

Cuadro 1 - Caracterización de los docentes entrevistados

\begin{tabular}{|l|l|l|l|}
\hline Entrevistado/a & \multicolumn{1}{|c|}{ Escuela } & \multicolumn{1}{|c|}{ Formación académica } & \multicolumn{1}{c|}{$\begin{array}{c}\text { Experiencias en investigación } \\
\text { científica }\end{array}$} \\
\hline Docente A & Gestión Pública & $\begin{array}{l}\text { Profesora en Cs. Naturales, con postítulo } \\
\text { en enseñanza de las Cs. Naturales, } \\
\text { graduada en un Instituto de Formación } \\
\text { Docente. }\end{array}$ & $\begin{array}{l}\text { No posee experiencias formales en } \\
\text { investigación científica. }\end{array}$ \\
\hline Docente B & Gestión Privada & $\begin{array}{l}\text { Profesor de Biología graduado en } \\
\text { Instituto de Formación Docente. }\end{array}$ & $\begin{array}{l}\text { Experiencias de investigación en } \\
\text { temáticas pedagógico-didácticas. }\end{array}$ \\
\hline Docente C & Gestión Pública & $\begin{array}{l}\text { Bióloga y Profesora en Cs. Biológicas, } \\
\text { graduada universitaria. }\end{array}$ & $\begin{array}{l}\text { Experiencias de investigación científica } \\
\text { en el campo disciplinar de la Biología. }\end{array}$ \\
\hline Docente D & Gestión Privada & $\begin{array}{l}\text { Bioquímica y Profesora en Bioquímica, } \\
\text { graduada universitaria }\end{array}$ & $\begin{array}{l}\text { Experiencias informales de investigación } \\
\text { disciplinar en Bioquímica. }\end{array}$ \\
\hline
\end{tabular}

Fuente: elaborado por los autores.

Previo a su utilización, el instrumento fue sometido a una prueba piloto con una docente jubilada del mismo espacio curricular, con el objetivo de analizar el potencial del guión para obtener información relevante para la investigación, así como también evaluar si las preguntas eran sencillas de comprender y si el orden de estas favorecía el diálogo con el entrevistado/a. A partir de esta prueba se realizaron algunas modificaciones que dieron lugar a la versión final del instrumento. 
La entrevista se organizó en dos partes. La primera indaga sobre aspectos generales de los contenidos y las actividades que cada docente lleva a cabo en el espacio curricular MICN, como así también acerca de la opinión del entrevistado/a sobre cuál eje de la NOS es el que prioriza en la enseñanza. Para esta pregunta, se mostró en papel a los entrevistados/as un esquema sobre el cual se les solicitó que seleccionaran entre los ejes epistemológico, sociológico e histórico de la NOS, presentados en forma de tres interrogantes: ¿qué es la ciencia y cómo se elabora? (eje epistemológico), ¿cómo se relaciona la ciencia con la sociedad y la cultura? (eje sociológico), y ¿cómo cambian las teorías científicas a lo largo del tiempo? (eje histórico) (ADÚRIZ-BRAVO, 2005).

La segunda parte de la entrevista indaga específicamente acerca de las concepciones y prácticas de enseñanza declaradas sobre sociología de la ciencia. Para confeccionar el listado de preguntas de esta sección se utilizaron como referencia algunas de las cuestiones de sociología externa e interna de la ciencia incluidas en el COCTS (VÁZQUEZ ALONSO et al., 2006). En cuanto a la sociología externa, se incluyeron preguntas acerca de las concepciones sobre la influencia de la sociedad sobre la ciencia, de la ciencia sobre la sociedad y de la política sobre la ciencia. Respecto de la sociología interna, se indagaron las concepciones acerca de las características personales del científico/a, y los efectos de las diferencias de género en la actividad científica. Luego, con el objetivo de indagar en las prácticas de enseñanza, para cada una de estas dimensiones se les consultó a los entrevistados/as si estos contenidos eran abordados en sus clases y de qué manera lo hacían.

En todos los casos, las preguntas fueron leídas de manera textual a cada docente y se realizaron repreguntas, aclaraciones o ampliaciones en las instancias en que se evaluó necesario. Las entrevistas fueron grabadas en audio, transcritas y luego sometidas a un proceso de paráfrasis. En una primera etapa de análisis se suprimieron aquellas secciones menos relevantes o que tenían repeticiones y luego se condensaron aquellos resultados similares. La paráfrasis del contenido de las entrevistas fue utilizada como un recurso que permitió un análisis cualitativo de los resultados, de una manera coherente y concisa, con base en la literatura previa (MIRANDA; FREITAS, 2008). Cabe destacar que el objetivo del análisis no consiste en describir el pensamiento individual de cada docente, sino en identificar tendencias en la comprensión del grupo de entrevistados/as, cuya discusión y problematización resultan relevantes en el marco de la investigación acerca de la enseñanza de la NOS.

\section{Resultados y Discusión}

\section{Aspectos Generales de la Enseñanza de la NOS en el Espacio Curricular MICN}

Las respuestas de los cuatro docentes entrevistados/as indican que en el diseño del currículo de MICN existe una priorización de los contenidos de tipo epistemológico. Se le otorga un lugar predominante a la enseñanza de los conceptos de ciencia y método científico y a la diferenciación del conocimiento científico respecto a otros tipos de saberes. Junto a estos conceptos, se trabajan contenidos procedimentales, tales como la formulación de problemas e hipótesis y la construcción de marcos teóricos. Además de ocupar la mayor porción del currículo, estos contenidos son los elegidos por este grupo de docentes para comenzar el ciclo lectivo: 
Comenzamos viendo qué es la ciencia y qué idea traen ellos sobre ciencia. [Docente A].

En cuarto año se enseñan contenidos epistemológicos: método científico, tipos de conocimiento, formulación de problemas e hipótesis. [Docente C].

Uno de los primeros contenidos fue la relación entre ciencia y pseudociencias. Se distribuyó a cada estudiante alguna pseudociencia y tenían que investigar de qué se trataba y cómo se podía justificar o refutar desde el conocimiento científico. [Docente B].

En particular, este último fragmento muestra que cuestiones tales como la demarcación del conocimiento científico respecto de las pseudociencias, en las cuales sería relevante incorporar referencias a aspectos sociológicos de la NOS (ZEMPLÉN, 2007), son abordadas desde un enfoque principalmente epistemológico.

Las opiniones de los docentes entrevistados/as coinciden con las obtenidas por Aikenhead (2006), quien investigó acerca de los contenidos de NOS desarrollados por docentes de ciencias y encontró que la mayoría de los profesores están motivados principalmente por enseñar los principios de la ciencia, lo que Jenkins (2002, p. 20) Ilama "[...] la lealtad de los docentes de secundaria a la disciplina".

Otro aspecto en el que coinciden todos los entrevistados/as radica en que el tratamiento de estos principios epistemológicos se considera como una base que sostiene el posterior trabajo de los y las estudiantes en un proyecto de investigación escolar: "Las chicas [las estudiantes] hacen un proyecto de investigación científica escolar, tienen que aplicar todo lo que vimos cuando trabajamos método, desde el armado del marco teórico, el planteo del problema y el planteo de la hipótesis." [Docente D].

Según Acevedo-Díaz y García-Carmona (2016), la realización de indagaciones científicas escolares es uno de los contextos típicos en los cuales se pone en juego la enseñanza de la NOS. Esta estrategia prioriza la enseñanza de cuestiones epistemológicas y metodológicas, tales como las influencias mutuas entre la formulación de la pregunta de investigación y las metodologías utilizadas para obtener resultados.

Asimismo, en todos los casos el proyecto de investigación escolar culmina con la redacción de un informe escrito por parte de los y las estudiantes, que en su estructura se asemeja a la manera en la que se presentan los artículos científicos:

Después traen todos los datos con los que tienen que hacer un informe, donde lo que más se les evalúa es la capacidad que han tenido para recoger la información, obtener resultados y analizarlos. [Docente A]. Vemos cómo es la estructura de un informe, que en el informe se vean reflejados los pasos del método científico, cómo se debe citar la bibliografía. [Docente C].

A pesar de que, como se evidencia, las temáticas epistemológicas adquieren el lugar protagónico en el currículo de MICN, los docentes entrevistados/as manifiestan incluir también contenidos y actividades vinculadas a los ejes histórico y sociológico de la NOS. En tres casos se incluyen contenidos específicos acerca de las transformaciones en las concepciones de ciencia a lo largo de la historia. En cuanto a los contenidos sociológicos, siguiendo lo propuesto por Bennásar Roig et al. (2010), en tres casos encontramos un tratamiento explícito y en uno hay un tratamiento implícito, transversal a otros contenidos.

En otra instancia de la entrevista, se presentó a los entrevistados/as un esquema que sintetiza tres grandes preguntas acerca de la ciencia, los cuales corresponden, según Adúriz-Bravo (2005), a los ejes en los que pueden clasificarse los diversos contenidos incluidos dentro de la NOS: el epistemológico, el sociológico 
y el histórico. Ante la pregunta acerca de cuál de estas cuestiones consideraban que describe mayormente las actividades y los contenidos de su espacio curricular, los cuatro docentes entrevistados/as seleccionaron la pregunta correspondiente al eje sociológico.

Este resultado es Ilamativo, debido a que el análisis de las respuestas anteriores indicó que los contenidos desarrollados con mayor extensión y profundidad corresponden a cuestiones epistemológicas. Sin embargo, un resultado similar fue encontrado por Aikenhead (2006), quien indagó a docentes acerca de si consideraban apropiado utilizar una perspectiva sociológica en la enseñanza de las ciencias. Frente a esta pregunta, más del $90 \%$ de los entrevistados afirmó que les parecía muy relevante, aunque luego la mayoría no lo implementaba realmente en sus clases.

Es interesante, entonces, recuperar y analizar los fragmentos de las entrevistas en los cuales los docentes entrevistados/as brindan una justificación frente a su elección del eje sociológico. Tanto la docente $A$ como la $D$ fundamentan que los asuntos vinculados a la sociología de la ciencia son tratados habitualmente en el diálogo áulico, en tanto coinciden con interrogantes que el propio estudiantado presenta acerca de la actividad científica:

El tema de la incidencia en la cultura, en la sociedad, te lo sacan ellas solas. Porque hay tanto en la actualidad donde los productos de la ciencia generan discusiones. [Docente D].

Los chicos [los estudiantes] se preguntan por ese tema. Por ejemplo, sobre salud, ¿Por qué no sale la vacuna para tal enfermedad?, ¿Por qué si saben que hace mal se sigue utilizando? [Docente A].

Desde otra perspectiva, el docente $B$ sostiene que ubica a la enseñanza del espacio curricular MICN dentro de una finalidad global del sistema educativo, que en su opinión consiste en crear vinculaciones entre los contenidos disciplinares y el contexto social y cultural. De allí el énfasis que intenta dar al eje sociológico:

El nombre de la materia es Metodología de la Investigación en Ciencias Naturales y me parece que es muy cerrado el concepto que tiene la materia en sí, el objetivo es más abarcativo, que los chicos [los estudiantes] puedan entender al conocimiento científico no como algo que se trabaja solamente en un laboratorio [...] Creo que va más en relación a lo social y lo cultural, porque está más relacionado a lo educativo, o sea, yo lo pienso desde la educación. [Docente B].

Estas diversas intencionalidades coinciden en gran medida con lo descrito por Auler y Delizoicov (2006) respecto a las finalidades que persigue el enfoque CTS en la didáctica de las ciencias. Es así que pueden encontrarse posiciones que proponen contemplar el análisis de estas interacciones sólo como un factor de motivación para el estudiantado, mientras que otras opiniones postulan que el entendimiento y el pensamiento crítico acerca de estos aspectos es un elemento esencial en la educación científica.

En síntesis, al consultar a los docentes entrevistados/as cuáles son los contenidos que seleccionan y las estrategias de enseñanza que proponen en MICN, surgen en primer lugar y con mayor extensión los aspectos epistemológicos. Sin embargo, al hacer visible una mirada más global y holística de la NOS, se evidencia también el tratamiento de temáticas sociológicas en el aula. 


\section{Concepciones y Prácticas de Enseñanza declaradas acerca de Aspectos de la Sociología Externa e Interna de la Ciencia}

\section{Sociología externa: relaciones entre la ciencia, la sociedad y la política}

Al indagar al grupo de docentes acerca de si la ciencia influye sobre la sociedad, en todos los casos afirman que esta influencia efectivamente existe, pero difieren en la argumentación acerca de la manera en la cual ésta se hace visible. Dos docentes coinciden en que la ciencia influye sobre la sociedad debido a que la intencionalidad subyacente a la construcción de conocimiento sería la búsqueda de soluciones a problemáticas y mejoras en la calidad de vida de la población:

\footnotetext{
Totalmente, [la ciencia influye sobre la sociedad porque] la ciencia es parte de la sociedad y obviamente lo que busca la ciencia es el conocimiento en sí mismo para mejorar la calidad de vida de las personas. [Docente C].

La ciencia evidentemente influye. Habitualmente responde a solucionar problemas que son propuestos por los grupos que ponen el dinero para financiar eso. Tiene un cierto condicionamiento de quién pone el dinero, porque lamentablemente necesita el dinero. No obstante, yo creo que aún con ese condicionante, tiene más de beneficioso que de perjudicial. [Docente D].
}

Estas opiniones pueden asimilarse a lo que Auler y Delizoicov (2006) denominan como la perspectiva salvacionista/redentora de la ciencia y la tecnología. Puede sintetizarse en la idea acerca de que los problemas actualmente existentes, así como también los que ocurrirán en el futuro, serán resueltos mediante un mayor desarrollo de la ciencia y la tecnología. También, en la visión según la cual, a medida que tengamos más ciencia y más tecnología, tendremos "un final feliz para la humanidad" (AULER; DELIZOICOV, 2006, p. 343).

Por otro lado, en los otros dos casos se hace hincapié en el hecho de que la construcción del conocimiento científico ejerce, por sí misma, un efecto sobre el entorno social. El docente $B$ se refiere en este sentido a la difusión en la sociedad de información proveniente de la investigación científica - que puede ser verídica o no y cómo ésta permea en la agenda de la opinión pública:

Yo creo que estamos todo el tiempo bombardeados por lo que se hace en ciencia, que no sabemos si es realmente cierto, porque uno ve Facebook y hay avances. ¿Qué información de la que se habla sobre el desarrollo del conocimiento científico es cierta? Porque está lleno de información, 'se descubrió la cura contra el cáncer', hay diez mil publicaciones que hablan sobre eso, y probablemente ninguna sea cierta, o a lo mejor sí. Entonces sí influye en la sociedad, porque después eso repercute, se empieza a expandir por todos lados. [Docente B].

Opiniones similares, en cuanto a la importancia de las telecomunicaciones y de la información que circula en internet como medios a través de los cuales ocurre la influencia de la ciencia y la tecnología sobre la sociedad, fueron vertidas por algunos de los docentes entrevistados por Miranda y Freitas (2008).

Al indagar a los entrevistados/as específicamente acerca del rol que la ciencia cumple, o debería cumplir, frente a problemáticas sociales y ambientales de la actualidad, encontramos dos argumentaciones diferenciadas. Por una parte, una función de la ciencia sería la de generar conocimiento sobre una determinada 
problemática y comunicar esa información a la sociedad con el objetivo de generar concientización:

El rol de la ciencia es justamente el de dar pruebas de las consecuencias que pueden traer algunas actividades sobre la calidad de vida de las personas, por ejemplo. [Docente A].

Para mí el primer rol es el de informar a la población, que después necesitará una concientización, porque me parece que sobre muchas temáticas hay información, pero lo que no hay es concientización, eso ya sería una segunda instancia. [Docente D].

Por otro lado, otra función de la ciencia consistiría en elaborar y aportar soluciones concretas a dichas problemáticas:

Pienso que todo el tiempo la ciencia está aportando a mejorar las problemáticas ambientales, de contaminación, de todo tipo. [Docente B].

Me parece que la principal función de la ciencia es aportar a los gobiernos soluciones concretas para esos problemas. Creo que los gobiernos las buscan, me parece que cuando necesitan solucionar problemas, yo supongo que recurrirán a los científicos. [Docente D].

Autores actuales dentro de la filosofía y la sociología de la ciencia, como Javier Echeverría y Jorge Linares, sostienen que a partir de la Segunda Guerra Mundial con el Proyecto Manhattan como hito - se consolida una nueva modalidad de hacer ciencia, a la cual denominan como tecnociencia. Entre los elementos distintivos de la tecnociencia se destacan una fuerte vinculación con el ámbito empresarial, una pluralidad de agentes vinculados a la toma de decisiones sobre las agendas de investigación y una racionalidad pragmático-utilitaria (ECHEVERRÍA, 2003; LINARES, 2008; PALLITO; DI PASQUO, 2017). Según esta racionalidad, el objetivo de la ciencia es modelar, diseñar, producir y manipular los objetos del mundo, descubriendo posibilidades operativas para dominar y controlar la naturaleza. Esta perspectiva viene a reemplazar a la racionalidad logocéntrica, asignada tradicionalmente a la ciencia moderna, cuyos objetivos consisten en explicar y describir de forma teórica el mundo en el que vivimos. Sin embargo, ambas racionalidades, la pragmático-utilitaria de la tecnociencia y la logocéntrica de la ciencia tradicional, conviven en los sistemas científico-tecnológicos de la actualidad (LINARES, 2008; PALLITO; DI PASQUO, 2017).

Introducimos estas consideraciones a fin de resaltar que las diferentes concepciones vertidas por los docentes entrevistados/as acerca de las relaciones entre la ciencia y la sociedad pueden analizarse en correspondencia con cada una de estas dos racionalidades. Podemos decir que cuando sostienen que la ciencia busca solucionar problemas, mejorar la calidad de vida de las personas o responder a demandas sociales, se sitúan en la racionalidad pragmático-utilitaria. Mientras tanto, cuando se refieren a la producción de conocimiento por sí misma como principal finalidad de la ciencia, sus opiniones se corresponden con la racionalidad logocéntrica. Es posible concluir que ambas apreciaciones describen dos modalidades de hacer ciencia que efectivamente conviven, no sin tensiones, en la actualidad. Sin embargo, las ideas acerca de la ciencia como proveedora de soluciones a las problemáticas sociales y ambientales, que en ningún caso la ubican como parte del origen o de la dinámica de estos fenómenos, se emparentan con la perspectiva salvacionista/redentora mencionada anteriormente (AULER; DELIZOICOV, 2006), la cual constituye una concepción ingenua acerca de las relaciones entre la ciencia y la sociedad. 
En relación a las prácticas de enseñanza acerca de estas temáticas, tres docentes llevan a cabo un tratamiento explícito de estos contenidos. Tal es así que el docente $B$ expresa que existe una unidad del programa de MICN en la cual se tratan los "aspectos políticos, éticos, filosóficos y socio-económicos vinculados a la acción científica". Sin embargo, no detalla cuál es el tipo de actividades por medio de las cuales se enseña el contenido. Otros autores también encontraron que los y las docentes de ciencias otorgan una gran importancia al abordaje de los aspectos sociales de la ciencia en el aula, pero que, al mismo tiempo, les resulta dificultoso definir qué estrategias podrían ser aplicadas para promover la participación y la comprensión de estos contenidos por parte del estudiantado (KIST; FERRAZ, 2010).

Por su parte, las docentes $C$ y $D$ explicitan una estrategia para el tratamiento de estos contenidos, que consiste en el uso de cuestiones socio-científicas concretas como herramienta para la enseñanza de las relaciones ciencia-sociedad. En los dos casos se utilizan artículos científicos, periodísticos y de divulgación científica como recursos para llevar a cabo estas actividades:

Trabajamos cómo se informa en diarios y revistas, comparándolos con un informe científico, un paper. Lo hacemos en distintas temáticas: el veganismo, el uso de agroquímicos, el aumento de cáncer, la problemática de transgénicos, la clonación. [Docente C].

Las chicas [las estudiantes] eligen una temática y buscan artículos de divulgación y artículos de investigación científica, y se cotejan, porque como el artículo de divulgación está escrito, en el mejor de los casos, por un periodista científico, siempre está permeado por otras cuestiones que el científico no [...] Tratamos de leer todo y de hacer un balance y ver por qué intereses puede estar permeado cada uno. [Docente D].

Una lectura crítica y reflexiva de noticias científicas presentes en los diarios locales puede favorecer la comprensión de la NOS (CAKMAKCI; YALAKI, 2011; SHIBLEY JR., 2003). Según García-Carmona (2015), es importante que los y las docentes realicen una selección previa para analizar su adaptación al ámbito educativo y para identificar los posibles sesgos periodísticos o simplificaciones. Asimismo, el trabajo con noticias periodísticas le brinda al alumnado la capacidad de seguir aprendiendo ciencia a lo largo de toda la vida, ya que para gran parte de la población las noticias consisten en una de las principales fuentes de información acerca de los avances de la ciencia (HODSON, 2008).

En cuanto a la indagación acerca de la influencia de la política sobre la ciencia, el elemento común a todas las respuestas se refiere a la cuestión del financiamiento: "Lo que yo creo es que la principal incidencia que tiene la política es desde la parte económica, cuánto aporta o cuánto saca, cuánto deja de poner" [Docente D].

Dos docentes sugieren, de forma poco precisa, que puede haber otras vías de influencia de la política sobre la ciencia, que van más allá de la cuestión estrictamente económica. La docente C elabora una definición más amplia de la política desde una perspectiva de desconfianza, mientras que la docente $D$ denomina a dichos aspectos como "lo ideológico".

La sociedad es política. La política es todo, lamentablemente o no. Hoy en día, vos decís política y pienso en algo asqueroso, me dan ganas de vomitar. Pero la política es la relación que existe entre las personas, eso es política. [Docente C]. 
[...] No sé si después habrá, en algunos temas, alguna incidencia de lo ideológico. Lo que seguro está siempre es la parte económica, a algunos proyectos se les da más dinero y a otros se les saca, según las líneas que los Estados quieren apoyar. [Docente D].

Sin negar que el financiamiento juega un rol privilegiado en la definición de las agendas de investigación, es relevante evidenciar que la ciencia tiene una dimensión política que va aún más allá, y tiene que ver con el rol que se le asigne en el programa político de una sociedad concreta, el cual está moldeado sistemáticamente por poderes gubernamentales, comerciales, militares y eclesiásticos, entre otros (ZIMAN, 2003).

Los docentes entrevistados/as expresaron que no abordan la temática de la influencia de la política en la ciencia de manera explícita en MICN. Únicamente la docente A indicó que trabaja con sus estudiantes algunas reseñas sobre las instituciones que componen el sistema científico nacional. Coincidiendo con Irzik y Nola (2011), este tipo de contenidos tiene relevancia para la enseñanza de la NOS y está incluido dentro de la dimensión social-institucional del enfoque de parecido de familia.

\section{Sociología interna: características personales del científico/a}

En esta instancia de la entrevista pedimos a los docentes entrevistados/as que indicaran cuáles eran las primeras palabras o imágenes que se les ocurrían al pensar en un científico/a. Un elemento común que aparece en todas las respuestas es el considerar a los científicos/as como personas curiosas, que dedican mucho tiempo, dedicación y voluntad a su trabajo. Estas ideas pueden verse reflejadas en diversos aspectos de las visiones simplistas y deformadas de la ciencia descritas en la extensa revisión bibliográfica realizada por Fernández et al. (2002).

Más allá de estos elementos comunes, pueden analizarse dos concepciones diferenciadas en cuanto a la relación del científico/a con su entorno. Por un lado, el docente $B$ y la docente $C$ perciben al científico/a como una persona ensimismada en su trabajo, un tanto aislada de la sociedad y del contexto en el cual desarrolla su trabajo:

Veo a un científico y veo tiempo en el laboratorio, yo veo que el científico tiene poco contacto con la sociedad. [Docente B].

Un científico que triunfa o un científico que está enroscado en su trabajo, es todo el tiempo científico. Todo el tiempo está pensando, nunca deja de maquinar. Esa curiosidad, esa mente así... calculadora, está todo el tiempo a full. [Docente C].

El sociólogo de la ciencia Bruno Latour (LATOUR, 1992) hizo referencia al término "torre de marfil" para describir este estereotipo acerca de los científicos/as como personas aisladas, abstraídas, excesivamente dedicadas a su trabajo y sin ninguna preocupación en común con el resto de la sociedad.

Por el contrario, las docentes A y D consideran que los científicos/as tienen una preocupación por las vinculaciones de su actividad con la sociedad. Incluso, sostienen que su trabajo se guía por ideales y que tiene la finalidad de mejorar la calidad de vida de la población:

La primera palabra que se me viene a la cabeza es 'idealista'. En un principio, cuando una persona es joven, quiere cambiar muchas cosas. Una persona creativa, que piensa que la calidad de vida puede ser mejor. [Docente A]. 
Alguien preocupado por la sociedad. Yo creo que la mayoría de los científicos buscan progresos que tengan que ver con el bien común. Gente muy curiosa. En última instancia, yo creo que generosos, porque aunque muchos puedan buscar un beneficio personal, económico o un reconocimiento social, el aporte que hacen queda, los trasciende. [Docente D].

En el mismo sentido, un estudio de Manassero Mas y Vázquez Alonso (2001) entrevistó a docentes acerca de cuál consideraban que era la principal motivación de los científicos. Encontraron que una gran cantidad de profesores, al igual que las docentes $A$ y $D$, opinaban que la principal motivación era conocer y beneficiar a la sociedad.

En cuanto al abordaje de las características personales del científico/a en las clases de MICN, la docente $C$ declara trabajar explícitamente este contenido utilizando viñetas de humor gráfico sobre la personalidad del científico/a y cómo se abordan las dificultades que pueden ocurrir en su actividad:

Trabajé con una especie de chiste que me encantaba. Es un astrónomo, que se le viene todo abajo el observatorio, al tipo lo ves mal vestido, y después piensa, se le prende la lamparita y empieza a trabajar con el tema del observatorio y la astrología. Entonces empieza a promocionar la astrología, empieza a venir gente a verlo y cobra entradas. Cuando termina de reconstruir el observatorio vuelve a su trabajo de medir y demás. Cada estudiante tenía que analizar distintas caricaturas. [Docente C].

Según Gallego (2007), por lo general, los cómics han ofrecido una visión distorsionada y estereotipada de la ciencia, donde se refuerza una imagen individualista, elitista, sexista, y aislada de la sociedad y la tecnología. Sin embargo, si se realiza una adecuada elección del cómic y de la actividad áulica, pueden constituir un vehículo atractivo para comunicar ideas sobre la NOS (TATALOVIC, 2009).

Los otros docentes expresaron no llevar a cabo actividades en donde se desarrolle la temática de manera explícita. Dos docentes relataron que, a pesar de no estar planificado, en el diálogo áulico se generan reflexiones sobre este asunto, en donde se evidencian ideas previas del estudiantado sobre las características personales de los científicos/as, que consisten en considerarlos como entidades superiores, con características diferentes a las del resto de las personas:

En mis clases, cuando vemos el concepto de ciencia lo hacemos a partir de lo que los chicos [los estudiantes] reconocen como ciencia y científico. Ahí es cuando surge esto de que el científico no es alguien más de la sociedad, sino alguien que tiene características muy particulares y para las que ellos en general no se ven. Creen que no dan para esas condiciones. [Docente D].

El docente $B$ y la docente $A$ declaran que al tratar esta temática tienen la intención de desmitificar ciertas percepciones erróneas de los estudiantes sobre la personalidad del científico/a y sobre su vida laboral. Es interesante observar la reflexión de $B$, en la cual asume que su propia concepción del científico/a como un ser aislado de la sociedad está claramente vinculada a una idea estereotipada:

Si bien esa es mi percepción del científico, trato de desmitificar la percepción del científico tradicional que uno tiene. Si vos me ponés a mí a imaginarme al científico me lo imagino como Einstein, una cosa que cualquier chico imaginaría, pero en realidad yo trato de romper con ese estereotipo. [Docente B]. 
Queda abierto el interrogante acerca de si los docentes entrevistados/as logran facilitar la construcción en sus estudiantes de una visión del científico/a como alguien que forma parte de una sociedad, que no tiene características particulares que lo conviertan en una entidad superior y que existe una heterogeneidad de atributos e intencionalidades dentro de la comunidad científica, teniendo en cuenta que el análisis de sus concepciones sobre la personalidad de los investigadores/as demuestra que, aún con contrastes, se asientan en ideas estereotipadas e ingenuas.

\section{Sociología interna: efectos de las diferencias de género en la actividad científica}

Todos los entrevistados/as sostienen que no existe ninguna diferencia de capacidad entre científicas y científicos a la hora de llevar a cabo la actividad científica. Tres docentes reconocen que, sin embargo, prevalecen asimetrías entre varones y mujeres en cuanto a su situación laboral dentro del sistema científico, como así también respecto a la visibilidad que adquieren sus aportes a la investigación:

\footnotetext{
Si bien hay cada vez más mujeres, o siempre hubo mujeres que desarrollaron la actividad científica, no se ve, no llega a verse eso. [Docente A].

Reconozco que tanto a nivel del mundo científico como en otros espacios todavía prevalece el hecho del hombre sobre la mujer y cómo se les complica a las mujeres llegar a cargos altos. Pero yo, en lo personal, $y$ de hecho es lo que inculco en mis estudiantes, creo que no hay diferencias, no las hubo nunca. Lo que pasa es que estamos en una sociedad que todavía es machista. Entonces esta cuestión sigue estando. [Docente B].
}

La docente $C$ agrega una visión optimista sobre los cambios que ocurren en la actualidad, tendiendo a una mayor igualdad y visibilidad del trabajo de las científicas:

Yo creo que eso está cambiando ahora. Ahora se siente la voz de la mujer, incluso manifestada por hombres [...] Si vos hacés un análisis histórico de cómo nace la ciencia, dónde nace la ciencia, quiénes son los que hacían ciencia, no eran mujeres. Obviamente que el hecho de que a la ciencia la lleven a cabo científicas mujeres ha ido costando. Pero tenemos científicas de renombre, bastante, creo que cada vez va a haber más. [Docente C].

La visión optimista de la docente $C$ puede fundarse en el hecho de que en los últimos años existen iniciativas políticas de igualdad, una expansión de las oportunidades y cambios socioeconómicos en las relaciones de género, que han contribuido a aumentar el número de mujeres en el ámbito profesional. Sin embargo, una revisión de la literatura existente acerca de los estereotipos de género en ciencia reconoce que el problema está lejos de resolverse. Esto se ve reflejado en que una parte importante de la literatura reflexiona acerca de las iniciativas que se han puesto en marcha con el objeto de aumentar el interés en esta cuestión y reducir la brecha de género en la ciencia (VÁZQUEZ-CUPEIRO, 2015).

La posición de la docente $D$ difiere de las respuestas de los otros docentes en cuanto a que opina que en otros momentos históricos existían asimetrías en el trabajo científico de las mujeres respecto de los varones, pero que en la actualidad ya estarían superadas: "Yo supongo que ya hoy no hay diferencias [...] Siempre se dice que si sos mujer es como que todo te cuesta más, pero no sé si es algo que se sigue diciendo y era 
antes y ahora ya no es. Yo creo que ya no es tan así, me parece que en ningún ámbito, pero es una percepción mía". [Docente D].

En cuanto a las prácticas de enseñanza, el docente B declara que trabaja esta temática en el aula a través del relato de casos históricos en los que los aportes de las científicas fue invisibilizado y apropiado por científicos varones:

Trabajamos mucho en mis clases el rol de la mujer en la ciencia, cómo antiguamente la ciencia estaba destinada a varones y a algunas pocas mujeres que lo hacían de manera oculta, tapada por un hombre. Vemos diferentes casos, como el de Rosalind Franklin, en donde la mujer hizo el aporte, pero en realidad quien se visibilizaba era el hombre. [Docente B].

Por su parte, la docente $C$ propone una actividad que consiste en elaborar biografías de algún científico y de alguna científica, teniendo en cuenta que muchos de los y las estudiantes recordarán varones pero les será más difícil reconocer mujeres en ciencia:

En MICN tenemos especificamente la cuestión de trabajar con el tema de las mujeres en la ciencia. Está explícito. De hecho vamos a trabajar con biografías de científicos, que ellos redacten una biografía de los científicos, y tiene que ser una mujer y un hombre, una mujer sí o sí, no dos científicos masculinos. [Docente C].

En el mismo sentido que la docente C, Truffa (2012) propone un proyecto de enseñanza e investigación educativa para el estudiantado de nivel secundario que consideramos valioso a los fines de identificar los estereotipos de género presentes en la percepción de la ciencia de los y las adolescentes. La investigadora sugiere una actividad similar, ya que se le pide a los y las estudiantes que listen los científicos/as que conocen y luego reconozcan si existe disparidad en el número de varones respecto del de mujeres.

\section{Análisis General de las Concepciones de los Docentes Entrevistados/as}

Antes de concluir, resulta interesante elaborar una visión de conjunto acerca de las concepciones expresadas por los docentes entrevistados/as sobre diversos aspectos del eje sociológico de la NOS. En primera instancia, destacar que en todos los casos se reconocen las influencias mutuas entre la ciencia y la sociedad, alejándose así de una visión de ciencia aséptica y neutral. Sin embargo, se manifiestan varias concepciones ingenuas respecto a esta temática, en su mayor parte asimilables a la perspectiva salvacionista/redentora. Esto se observó tanto al preguntar sobre el modo en el que se relaciona la ciencia con la sociedad, como así también ante la indagación sobre cuál es el rol que juega la ciencia frente a problemáticas sociales y ambientales.

Otro aspecto en el cual es evidente la presencia de concepciones ingenuas es el vinculado a la imagen del científico/a, donde las opiniones expresadas por los docentes replican lo reportado en la literatura en cuanto a las visiones estereotipadas y simplistas acerca de la ciencia. Más aún, un entrevistado reconoce la controversia entre su propia imagen de los investigadores/as y aquella que busca construir con sus estudiantes. En contraste, el grupo de docentes manifiesta concepciones adecuadas acerca de un tema que cuenta actualmente con alta relevancia en el ámbito científico 
y en la sociedad en general, como es el debate acerca de las implicancias del género en la actividad científica.

\section{Consideraciones Finales}

El análisis de las entrevistas a docentes del espacio curricular MICN indica que persiste un énfasis en la enseñanza explícita del eje epistemológico de la NOS por sobre los ejes histórico y sociológico. Específicamente, en relación a la inclusión de los contenidos sociológicos, se encontró una heterogeneidad de respuestas, pero se evidenció que en la mayoría de los casos sí son trabajados en el aula. Las prácticas de enseñanza por medio de las cuales se tratan estas cuestiones son diversas. En algunos casos se utiliza un enfoque explícito y en otros casos se abordan de una manera espontánea, con un menor grado de planificación. Asimismo, puede observarse que estos asuntos no suelen ser priorizados en la sucesión de contenidos de la planificación anual del ciclo lectivo y que, en alguna medida, surgen como temáticas adicionales a los conceptos epistemológicos y a los procedimientos de elaboración de un proyecto de investigación escolar.

En este sentido, tal como plantean Kist y Ferraz (2010), a pesar de que los y las docentes reconocen la importancia de abordar estas temáticas, resulta complejo identificar explicaciones claras acerca de qué estrategias y prácticas utilizan para asegurar una mejor comprensión del tema y promover una participación activa en las discusiones en el aula y una visión crítica que impacte en la sociedad en general. Puede considerarse a este aspecto como un reflejo más del problema del consenso acerca de la NOS.

Por otra parte, las concepciones de los profesores entrevistados/as acerca de la sociología externa e interna de la ciencia resultaron ser, en algunos aspectos, informadas y adecuadas a los conocimientos actuales, mientras que en otros asuntos se evidenciaron perspectivas ingenuas. Asimismo, se manifestó también cierto grado de ambigüedad y contradicción en sus respuestas a las distintas cuestiones indagadas. Esta falta de claridad puede comprenderse si se tiene en cuenta la gran complejidad que implica el estudio de las relaciones entre la ciencia y la sociedad, teniendo en cuenta que estos aspectos pueden ser abordados y discutidos desde diversos ángulos. Es así que, a medida que el conocimiento científico y tecnológico es producido, se construyen también determinados discursos, formas de ver y analizar dicha producción, que son elaborados y diseminados por determinados actores sociales (AULER; DELIZOICOV, 2006). Estos discursos influyen en la definición del currículum escolar en ciencias, así como también en las concepciones de docentes y estudiantes acerca de la NOS.

Cabe destacar que esta investigación fue realizada en un espacio curricular que está orientado específicamente a la enseñanza de la NOS, por lo cual se convierte en un ámbito propicio para conocer las concepciones de los y las docentes, así como las prácticas de enseñanza acerca de los aspectos sociológicos de la ciencia, menos estudiados por la investigación en didáctica de las ciencias en comparación al eje epistemológico. Como proyección futura de nuestro trabajo, sería interesante continuar analizando estas cuestiones en profesores de los espacios curriculares de las distintas disciplinas científicas 
(biología, química, física, etc.). También se vuelve relevante profundizar en la realización de entrevistas con otro tipo de formulaciones. Por ejemplo, mediante la presentación de cuestiones sociocientíficas concretas, en donde se puedan evaluar los intereses e intencionalidades de distintos actores y consultar al entrevistado/a qué rol juega la ciencia en cada caso.

A modo de cierre, destacamos que la NOS constituye un componente esencial de la alfabetización científica de la ciudadanía. En particular, sostenemos que conocer los principios elementales del funcionamiento de la ciencia actual, tanto en sus características internas como en su relación con la sociedad, es un elemento básico para una educación científica que permita a las personas contar con información esencial para la toma de decisiones sobre nuestro modo de vida. Tal como plantea Matthews (2012), no es realista esperar que docentes y estudiantes sean expertos en historia, filosofía o sociología de la ciencia. El objetivo debe ser fomentar en docentes y estudiantes una visión menos compartimentalizada del conocimiento, propiciando que estas múltiples perspectivas se hagan visibles en el aula de ciencias.

\section{Referencias}

ACEVEDO-DÍAZ, J. A.; GARCÍA-CARMONA, A. "Algo antiguo, algo nuevo, algo prestado": tendencias sobre la naturaleza de la ciencia en la educación científica. Revista Eureka sobre Enseñanza y Divulgación de Las Ciencias, Cádiz, v. 13, n. 1, p. 3-19, 2016.

ADÚRIZ-BRAVO, A. Una introducción a la naturaleza de la ciencia: la epistemología en la enseñanza de las ciencias naturales. Buenos Aires: Fondo de Cultura Económica, 2005.

AIKENHEAD, G. S.; RYAN, A. G. The development of a new instrument: 'views on ScienceTechnology-Society' (VOSTS). Science Education, New York, v. 76, n. 5, p. 477-491, 1992. DOI: https://doi.org/10.1002/sce.3730760503.

AIKENHEAD, G. S. Science education for everyday life: evidence-based practice. Nueva York: Teachers College Press, 2006.

ALLCHIN, D. Teaching the nature of science: perspectives \& resources. Saint Paul: SHiPS Education Press, 2013.

ANTONIOLI, P. M.; CHRISPINO, A.; VÁZQUEZ ALONSO, A.; MANASSERO MAS, M. A. Evaluación de las actitudes de las dos culturas en relación al aprendizaje de la ciencia. Revista lberoamericana de Educación, Madrid, v. 58, p. 151-166, 2012. DOI: https://doi.org/10.35362/rie580478.

AULER, D.; DELIZOICOV, D. Ciência-tecnologia-sociedade: relações estabelecidas por professores de ciências. Revista Electrónica de Enseñanza de las Ciencias, Vigo, v. 5, n. 2, p. 337-355, 2006. Recuperado el 12 mar. 2021 de: http://reec.uvigo.es/volumenes/volumen5/ART8_Vol5_N2.pdf.

BEJARANO, N. R. R.; ADÚRIZ-BRAVO, A.; BONFIM, C. S. Natureza da ciência (NOS): para além do consenso. Ciência \& Educação, Bauru, v. 25, n. 4, p. 967-982, 2019. DOI: https://doi. org/10.1590/1516-731320190040008.

BENNÁSSAR ROIG, A.; VÁZQUEZ ALONSO, Á.; MANASSERO MAS, M. A.; GARCÍA-CARMONA, A. (coord.). Ciencia, tecnología y sociedad en Iberoamérica: una evaluación de la comprensión de la naturaleza de ciencia y tecnología. Madrid: Organización de Estados Iberoamericanos, 2010. Recuperado el 12 mar. 2021 de: https://idus.us.es/handle/11441/59306.

CAKMAKCI, G.; YALAKI, Y. Popular media as a tool for teaching science and its nature. In: PROMOTING teachers' ideas about nature of science through popular media. Ankara: Hacettepe University, 2011. p. 1-14. 
CÓRDOBA (Provincia). Secretaría de Educación. Diseño curricular de educación secundaria: orientación ciencias naturales 2012-2015. Córdoba: Gobierno de la Provincia, 2012. Recuperado el 7 jul. 2020 de: https://cutt.ly/YzIVMhv.

DAGHER, Z. R.; ERDURAN, S. Reconceptualizing the nature of science for science education: why does it matter? Science \& Education, Dordrecht, v. 25, n. 1, p. 147-164, 2016.

DÍAZ-BRAVO, L.; TORRUCO-GARCÍA, U.; MARTÍNEZ-HERNÁNDEZ, M.; VARELA-RUIZ, M. La entrevista, recurso flexible y dinámico. Investigación en Educación Médica, México, v. 2, n. 7, p. 162-167, 2013.

ECHEVERRÍA, J. La revolución tecnocientífica. Madrid: Fondo de Cultura Económica, 2003.

ERDURAN, S.; DAGHER, Z. R. Reconceptualizing nature of science for science education. Dordrecht: Springer, 2014.

FARÍAS, D. M. Teoría, estructura y modelos atómicos en los libros de texto de química de educación secundaria: análisis desde la sociología de la ciencia e implicaciones didácticas. 2012. 433 h. Tesis (Doctorado en Práctica Educativa y Comunicación) - Universidad de Barcelona, Barcelona, 2012.

FERNÁNDEZ, I.; GIL, D.; CARRASCOSA ALÍS, J.; CACHAPUZ, A.; PRAIA, J. Visiones deformadas de la ciencia transmitidas por la enseñanza. Enseñanza de las Ciencias, Barcelona, v. 20, n. 3, p. 477-488, 2002.

GALLEGO, A. P. Imagen popular de la ciencia transmitida por los cómics. Revista Eureka sobre Enseñanza y Divulgación de las Ciencias, Cádiz, v. 4, n. 1, p. 141-151, 2007.

GARCÍA-CARMONA, A. Noticias sobre temas de astronomía en los diarios: un recurso para aprender sobre la naturaleza de la ciencia reflexivamente. Revista de Enseñanza de la Física, Córdoba, Argentina, v. 27, n. 1, p. 19-30, 2015. Recuperado el 12 mar. 2021 de: https://revistas. unc.edu.ar/index.php/revistaEF/article/view/11408.

HODSON, D. Towards scientific literacy: a teachers' guide to the history, philosophy and sociology of science. Leiden: Brill Sense, 2008.

IRZIK, G.; NOLA, R. A. Family resemblance approach to the nature of science for science education. Science \& Education, Dordrecht, v. 20, n. 7, p. 591-607, 2011. DOI: https://doi. org/10.1007/s11191-010-9293-4.

IRZIK, G.; NOLA, R. New directions for nature of science research. In: MATTHEWS, M. (org.). International handbook of research in history, philosophy and science teaching. Dordrecht: Springer, 2014. p. 999-1021.

JENKINS, E. W. Linking school science education with action. Counterpoints, Berlin, v. 210, p. 17-34, 2002.

KIST, C. P.; FERRAZ, D. F. Compreensão de professores de biologia sobre as interações entre ciência, tecnologia e sociedade. Revista Brasileira de Pesquisa em Educação em Ciências, Belo Horizonte, v. 10, n. 1, p. 1-15, 2010.Norte, Natal, 2012.

LATOUR, B. Ciencia en acción. Barcelona: Labor, 1992.

LINARES, J. Ética y mundo tecnológico. México: Fondo de Cultura Económica, 2008.

LOZANO, E.; BAHAMONDE, N.; ADÚRIZ-BRAVO, A. Análisis histórico-epistemológico sobre los modelos de membrana celular para enseñar biología celular y naturaleza de la ciencia al profesorado. Filosofia e História da Biologia, São Paulo, v. 11, n. 1, p. 49-68, 2016.

MANASSERO MAS, M. A.; VÁZQUEZ, A. Actitudes de estudiantes y profesorado sobre las características de los científicos. Enseñanza de las Ciencias, Barcelona, v. 19, n. 2, p. 255-268, 2001. Recuperado el 12 mar. 2021 de: https://ddd.uab.cat/record/1529l. 
MATTHEWS, M. R. Changing the focus : from nature of science (NOS) to features of science (FOS). In: KHINE M.S. (org.). Advances in nature of science research. Dordrecht: Springer, 2012. p. 3-26.

MIRANDA, E. M.; FREITAS, D. A compreensão dos professores sobre as interações CTS evidenciadas pelo questionário VOSTS e entrevista. Alexandria, Florianópolis, v. 1, n. 3, p. 79-99, 2008.

MOURA, B. A. O que é natureza da ciência e qual sua relação com a história e filosofia da ciência. Revista Brasileira de História da Ciência, Rio de Janeiro, v. 7, n. 1, p. 32-46, 2014.

PALLITTO, N.; DI PASQUO, F. El espíritu (tecno) científico que convendría evitar y resistir. Ludus Vitalis, México, v. 25, n. 47, p. 261-264, 2017.

PUJALTE, A.; ADÚRIZ-BRAVO, A. A.; PORRO, S. De la imagen de ciencia declarativa a la de la práctica en el aula: las imágenes del profesorado entre la visión democrática y la deficitaria. Uni-Pluriversidad, Antioquia, v. 14, n. 2, p. 111-117, 2014. Recuperado el 12 mar. 2021 de: https:// revistas.udea.edu.co/index.php/unip/article/view/20062.

SEPINI, R. P.; MACIEL, M. D. Como o ensino de questões relacionadas com natureza da ciência e tecnologia pode contribuir para a formação de futuros professores. Indagatio Didactica, Aveiro, v. 8, n. 1, p. 739-751, 2016. DOI: https://doi.org/10.34624/id.v8i1.3478.

SHIBLEY JR., I. A. Using newspapers to examine the nature of science. Science \& Education, Dordrecht, v. 12, n. 7, p. 691-702, 2003. DOI: https://doi.org/10.1023/A:1025687424931.

TATALOVIC, M. Science comics as tools for science education and communication: a brief, exploratory study. Journal of Science Communication, Trieste, v. 8, n. 4, p. 1-17, 2009. DOI: https:// doi.org/10.22323/2.08040202.

TEÓFILO, F. B. S.; GALLÃO, M. I. História e filosofia da ciência no ensino de biologia celular. Ciência \& Educação, Bauru, v. 25, n. 3, p. 783-801, 2019. DOI: https://doi.org/10.1590/1516731320190030012.

TRUFFA, A. Ciencia y estereotipos de género: una propuesta de enseñanza e investigación en nivel secundario. In: JORNADAS DE ENSEÑANZA E INVESTIGACIÓN EDUCATIVA EN EL CAMPO DE LAS CIENCIAS EXACTAS Y NATURALES, 3., 2012, La Plata, Argentina. Actas [...]. Recuperado el 12 mar. 2021 de: http://www.memoria.fahce.unlp.edu.ar/trab_eventos/ev.3718/ev.3718.pdf.

VÁZQUEZ ALONSO, A.; ACEVEDO-DÍAZ, J.; MANASSERO MAS, M. A.; ACEVEDO ROMERO, P. Evaluación de los efectos de la materia CTS de bachillerato en las actitudes CTS del alumnado con una metodología de respuesta múltiple. Revista Eureka sobre Enseñanza y Divulgación de las Ciencias, Cádiz, v. 3, n. 3, p. 317-348, 2006.

VÁZQUEZ ALONSO, A.; MANASSERO MAS, M. A. Una taxonomía para facilitar la enseñanza explícita de la naturaleza de la ciencia y su integración en el desarrollo del currículo de ciencias. Interacções, Santarém, Portugal, v. 11, n. 34, p. 312-349, 2015.

VÁZQUEZ ALONSO, A.; MANASSERO MAS, M. A. Interdisciplinariedad y conceptos nómadas en didáctica de la ciencia: consecuencias para la investigación. Revista Eureka sobre Enseñanza y Divulgación de las Ciencias, Cádiz, v. 14, n. 1, p. 24-37, 2016. Recuperado el 12 mar. 2021 de: https://revistas.uca.es/index.php/eureka/article/view/3003.

VÁZQUEZ-CUPEIRO, S. Ciencia, estereotipos y género: una revisión de los marcos explicativos. Convergencia, Toluca, Mexico, v. 22, n. 68, p. 177-202, 2015.

ZEMPLÉN, G. A. Putting sociology first: reconsidering the role of the social in 'nature of science' education. Science \& Education, Dordrecht, v. 18, n. 5, p. 525-559, 2007.

ZIMAN, J. Ciencia y sociedad civil. Revista Iberoamericana de Ciencia, Tecnología y Sociedad, Buenos Aires, v. 1, n. 1, p. 177-188, 2003. Recuperado el 12 mar. 2021 de: https://www.redalyc. org/pdf/924/92410110.pdf. 